\title{
Comparative Study of Active Control of a Large Composite I-Beam*
}

\author{
Gangbing Song and Vineet Sethi \\ Department of Mechanical Engineering, University of Houston, Houston, TX 77204 USA
}

(Received 14 April 2003; accepted 10 October 2003)

\begin{abstract}
This paper presents comparative experimental results of various active vibration control methods of a pultruded fibre-reinforced polymer (FRP) composites thin-walled I-beam using smart sensors and actuators. The FRP I-beams are made of E-glass fibers and polyester resins. The FRP I-beam is in a cantilevered configuration. PZT (Lead zirconate titanate) type of piezoelectric ceramic patches are used as smart sensors and actuators. These patches are surface-bonded near the cantilevered end of the I-beam. Utilising results from open-loop experimental testing, several active vibration control methods, such as positive position feedback (PPF), strain rate feedback (SRF), lead compensator and pole placement control are investigated. A comparative study and analysis of the control results obtained from the experiment was done. The study showed that the PPF control provides better vibration suppression than the SRF, lead compensator or pole placement control. Pole placement control results were superior to lead control, and SRF provided intermediate results. Experimental results demonstrate that the proposed methods achieve effective vibration control of the FRP I-beam.
\end{abstract}

* Sponsored by National Science Foundation via a career grant and NASA via a cooperative grant

\section{INTRODUCTION}

In recent years, fibre reinforced polymers have had spectacular applications in civil engineering structures. However, vibration suppression of these objects is also a critical issue. There is extensive literature available on vibration suppression methods that have been used in control of lightweight flexible structures. Substantial details of applications of smart materials for civil structures are included in reference 1. Piezoelectric materials have been used as actuators and sensors in flexible lightweight beams for passive and active damping. Passive damping using piezoelectric material has been studied by Hagood and Flotow, ${ }^{2}$ and active damping using piezoelectric actuators and sensors has been investigated by Sulla et al., ${ }^{3}$ Shen, ${ }^{4}$ Han et al., ${ }^{5}$ Sugavanam et al. ${ }^{6}$ and Blanguernon et al. ${ }^{7}$. Aizawa et al., ${ }^{8}$ Fujita et al. ${ }^{9}$ and Kamada et al. ${ }^{\mathbf{1 0}}$ have used piezoelectric stack actuators for active damping where the control force requirement is high, such as the response control of four storey structural frames. Installation of piezoelectric stack actuators requires some modification in the structure, whereas piezoelectric patch actuators can be surface bonded to the structure with or without minimal modification of the original structure.

Furthermore, in studies related to lightweight structures, there is no data available which cites the structure to actuator weight ratio. However, in this study, the structure to actuator weight ratio is 186 , which we believe is substantially higher than that of the lightweight flexible structures that also used the piezoelectric patch actuators. Hence, without any alterations to the structure, this work employs surface-bonded piezoelectric patch actuators of $(10.16 \times 3.81 \times 0.08) \times 10^{-2}$ centimetres in size and $58 \times 10^{-3} \mathrm{~kg}$ in weight to actively control vibrations of a 3.3-metre-long pultruded fibre-reinforced polymer (FRP) composite thin-walled I-beam structure that weighs $10.780 \mathrm{~kg}$. It is also to be noted that active control systems involve power amplifiers and other hardware, the weight of these is not considered while evaluating the structure to actuator weight ratio as 186 .

The FRP I-beam, made of E-glass fibres and polyester resins, is set up in a cantilevered configuration. Four active vibration control methods, positive position feedback (PPF), strain rate feedback (SRF), lead compensation and pole placement are designed and implemented. Positive position feedback is applied by feeding the structural position coordinate directly to the compensator, and the product of the compensator and a scalar gain positively back to the structure. ${ }^{11-14}$ PPF offers quick damping for a particular mode provided that the modal characteristics are known. PPF is also easy to implement. Song et al. ${ }^{15}$ experimentally demonstrated that PPF is insensitive to a varying modal frequency. SRF control is used for active damping of a flexible space structure. ${ }^{16}$ Using $\mathrm{SRF}$, the structural velocity coordinate is fed back to the compensator and the compensator position coordinate multiplied by a negative gain is fed back to the structure. SRF has a wider active damping region and can stabilise more than one mode, given a sufficient bandwidth. In this research, the $\mathrm{SRF}$ is designed to control the vibration of the 1 st mode. A lead compensator, if designed properly, can increase the damping ratio of a targeted mode by relocating the corresponding dominant pole. Manning et al. ${ }^{17}$ and Scott et al. ${ }^{18}$ used pole placement control for flexible beams. Experimental results demonstrate that the PPF, SRF, lead compensation and pole placement methods are effective in actively increasing damping of the flexible composite I-beam with PZT sensors and actuators.

\section{EXPERIMENTAL SETUP}

The control objective is to show the effectiveness of various vibration suppression strategies for a composite I-beam by using smart sensors and actuators. To achieve this control objective, an experiment is set up. A cantilevered composite I-beam is mounted on a structure and piezo patches are at- 